\title{
Early patency rate and fate of reattached intercostal arteries after repair of thoracoabdominal aortic aneurysms
}

\author{
Atsushi Omura, MD, Katsuhiro Yamanaka, MD, Shunsuke Miyahara, MD, Toshihito Sakamoto, MD, \\ Takeshi Inoue, MD, Kenji Okada, MD, and Yutaka Okita, MD
}

\begin{abstract}
Objectives: The present study analyzes the early patency of intercostal artery reconstruction, using graft interposition and aortic patch anastomosis, and determines the fate of reattached intercostal arteries after repair of thoracoabdominal aortic aneurysms.
\end{abstract}

\begin{abstract}
Methods: We selected 115 patients (mean age, $63 \pm 15$ years; range, 19-83 years; male, $n=83$ ) treated by thoracoabdominal aortic aneurysm repair with 1 or more reconstructed intercostal arteries at the Kobe University Graduate School of Medicine between October 1999 and December 2012. The intercostal arteries were reconstructed using graft interposition $(n=66)$, aortic patch anastomosis $(n=42)$, or both $(n=7)$.
\end{abstract}

\begin{abstract}
Results: The hospital mortality rate was $7.8 \%(n=9)$. Eleven patients $(9.6 \%)$ developed spinal cord ischemic injury (permanent, $\mathrm{n}=6$, transient, $\mathrm{n}=5$ ). The average number of reconstructed intercostal arteries per patient was $3.0 \pm 1.5(1-7)$, and 345 intercostal arteries were reattached. The overall patency rate was $74.2 \%(256 / 345)$ and that of aortic patch anastomosis was significantly better than that of graft interposition $(90.8 \%[109 / 120]$ vs $65.3 \%$ [147/225], $P<.01$ ), but significantly worse for patients with than without spinal cord ischemic injury $(51.9 \%$ [14/27] vs $76.1 \%$ [242/318], $P=.01)$. There was no patch aneurysm in graft interposition during a mean of $49 \pm 38$ (range, 2-147) postoperative months, but aortic patch anastomosis including 4 intercostal arteries became dilated in 2 patients.
\end{abstract}

Conclusions: Aortic patch anastomosis might offer better patency rates and prevent spinal cord ischemic injury compared with graft interposition. Although aneurysmal changes in intercostal artery reconstructions are rare, large blocks of aortic wall reconstruction should be closely monitored. (J Thorac Cardiovasc Surg 2014;147:1861-7)

Thoracoabdominal aortic aneurysm (TAAA) repair is a highly invasive procedure with 30 -day mortality rates ranging from $7.0 \%$ to $10.0 \%$ even at centers with high surgical volumes, ${ }^{1-3}$ and spinal cord ischemic injury (SCII) remains one of the most serious complications, occurring at an incidence of $3 \%$ to $5 \% \cdot{ }^{1-3}$ Despite advances during the past 2 decades, a reliable method of preventing SCII has not been established. Multidisciplinary approaches are important for the safe repair of TAAA, and revascularization of the intercostal arteries (ICAs) represents an important technical step. Despite much individual diversity, the spinal cord is usually supplied with blood from branches of the vertebral, internal iliac, and ICAs. Many surgeons have attempted to reattach ICAs during TAAA repair based on anatomic blood feeds. However, little is known about the patency rates of reattached ICAs and the clinical association with SCII.

\footnotetext{
From the Department of Cardiovascular Surgery, Kobe University Graduate School of Medicine, Kobe, Japan.

Disclosures: Authors have nothing to disclose with regard to commercial support. Received for publication Feb 20, 2013; revisions received April 22, 2013; accepted for publication June 27, 2013; available ahead of print Sept 16, 2013.

Address for reprints: Yutaka Okita, MD, Department of Cardiovascular Surgery, Kobe

University Graduate School of Medicine, 7-5-2 Kusunoki-cho, Chuo-ku, Kobe,

Hyogo 630-0005, Japan (E-mail: yokita@med.kobe-u.ac.jp).

$0022-5223 / \$ 36.00$

Copyright (c) 2014 by The American Association for Thoracic Surgery

http://dx.doi.org/10.1016/j.jtcvs.2013.06.035
}

Some authors think that ICA reattachment might be unnecessary because various collateral networks, such as branches of the subclavian or internal iliac arteries, also supply blood to the spinal cord. ${ }^{4}$ Late complications of reattached ICAs include the development of aneurysm. ${ }^{5}$ The clinical validity of ICA reattachment remains controversial. We aggressively reattach ICAs to maintain the blood supply to the spinal cord during TAAA repair at Kobe University Graduate School of Medicine. We investigated the patency rate, the relationship between the patency of reconstructed ICAs and SCII, and the fate of reattachment aortic intercostal patch during follow-up.

\section{MATERIALS AND METHODS}

All patients had previously granted permission for use of their medical records regarding information about preoperative status, surgery performed, postoperative course, medical examinations, and late follow-up for medical purposes. Patient safety and privacy were strictly managed in keeping the medical records. The institutional review board at Kobe University Graduate School of Medicine approved this retrospective observational review of the data collected for this study. Among 158 patients who underwent TAAA repair between October 1999 and December 2012 at the Kobe University Graduate School of Medicine, 131 required 1 or more ICA reconstructions. Of these, 1 patient with Crawford extent IV and 15 patients in whom reconstructed ICA patency could not be postoperatively evaluated by angiography or computed tomography $(\mathrm{CT})(\mathrm{n}=15)$ were excluded from the analysis. We finally analyzed data derived from 115 patients (male, $\mathrm{n}=83$ [72.2\%]; mean age, $63 \pm 15$ [range, 19-83] years). We classified TAAA as Crawford extent I $(\mathrm{n}=19)$, II $(\mathrm{n}=40)$, and III $(\mathrm{n}=56)$, and 


$$
\begin{aligned}
& \text { Abbreviations and Acronyms } \\
& \begin{aligned}
\text { AKA } & =\text { Adamkiewicz artery } \\
\text { CSF } & =\text { cerebrospinal fluid } \\
\text { CT } & =\text { computed tomography } \\
\text { ICA } & =\text { intercostal artery } \\
\text { SCII } & =\text { spinal cord ischemic injury } \\
\text { TAAA } & =\text { thoracoabdominal aortic aneurysm }
\end{aligned}
\end{aligned}
$$

21 patients had Marfan syndrome. A history of aortic surgery was defined as previous surgery to treat a descending thoracic or abdominal aortic aneurysm. Chronic renal failure was defined as serum creatinine $2.0 \mathrm{mg} / \mathrm{dL}$ or greater. Chronic obstructive pulmonary disease was defined as forced expiratory volume in 1 second less than $75 \%$ of the value predicted by spirometry. We preoperatively confirmed that 85 patients had an Adamkiewicz artery (AKA) by $\mathrm{CT}$ or magnetic resonance imaging angiography. Table 1 shows the preoperative characteristics of the patients.

\section{Surgical Technique}

The details of our technique for TAAA repair have been reported. ${ }^{6} \mathrm{We}$ usually placed a cerebrospinal fluid (CSF) drainage catheter into the subarachnoid space on the day before the surgery. For various reasons, such as emergency surgery, hemodynamic instability, coagulopathy, anatomic reasons, and drainage catheter occlusion, CSF drainage was accomplished in $82.6 \%$ of patients $(95 / 115)$. The CSF was allowed to freely drain whenever CSF pressure exceeded $10 \mathrm{~mm}$ Hg. Transcranial motor-evoked potentials were applied to monitor spinal cord ischemia during the procedure. The patients were intubated with a double-lumen endotracheal tube to allow the left lung to collapse. Heparin was administered to achieve an activated clotting time of greater than 400 seconds. Cardiopulmonary bypass was established via cannulation of the left femoral artery and the right femoral vein, and if necessary, the pulmonary artery was also cannulated for venous drainage. The pump circuit had an extracorporeal membrane oxygenator including a heat exchanger. Body temperature was usually set to between $31^{\circ} \mathrm{C}$ and $34^{\circ} \mathrm{C}$ (rectal temperature, $\mathrm{n}=78$ ). Deep hypothermia and circulatory arrest were applied if crossclamping of the proximal aorta seemed impossible $(\mathrm{n}=37)$. The aorta was anastomosed to a gelatin-impregnated woven Dacron graft. The aorta was sequentially clamped, and patent intercostal and lumbar arteries at the Th8 to L2 level were reimplanted by graft interposition or aortic patch anastomosis (Figure 1) and immediately reperfused. Graft interposition means that patent orifices of ICAs are reattached through a short graft $(8$ or $10 \mathrm{~mm}$ ) with an end-to-side anastomosis. Aortic patch anastomosis means that patent orifices of ICAs are anastomosed to a side hole on the graft as an aortic cuff using an inclusion technique. Significant back-bleeding from ICAs was controlled by inserting balloon catheters or by externally clamping the ICAs to alleviate the steal effect from the anterior spinal artery. Our intraoperative decision-making regarding which ICAs to reattach was based on the following findings. If an AKA was included in the range of graft replacement, we always tried to reconstruct the ICA that was considered as a donor to the AKA. In addition to AKA reconstruction, among the ICAs from Th8 to L2, we generally select ICAs for reattachment that have large orifices or those in which back-bleeding from the orifice is significant. If the amplitude of transcranial motor-evoked potentials declined, the ICA and lumbar artery were reattached as soon as possible. Mean distal perfusion pressure was always maintained at greater than $70 \mathrm{~mm} \mathrm{Hg}$. Visceral arteries were routinely perfused by selective cannulation using a separate roller pump to reattach the visceral arteries. Blood flow in each visceral branch was controlled at $150 \mathrm{~mL} / \mathrm{min}$. The visceral and renal arteries were separately reconstructed

\begin{tabular}{|c|c|c|c|c|c|}
\hline Variable & Overall $(\%)$ & Graft interposition $(\%)$ & Aortic patch anastomosis (\%) & Both methods (\%) & $P$ value* \\
\hline Patients & 115 & 66 & 42 & 7 & \\
\hline Age $($ mean $\pm \mathrm{SD})$ & $63 \pm 15$ & $62 \pm 16$ & $63 \pm 14$ & $65 \pm 12$ & .89 \\
\hline Male gender & $83(72.2)$ & $48(72.7)$ & $31(73.8)$ & $4(57.1)$ & .90 \\
\hline \multicolumn{6}{|l|}{ Aortic disease } \\
\hline Nondissection & 53 & $32(48.5)$ & $19(45.2)$ & $2(28.6)$ & .93 \\
\hline Dissection & 62 & $34(51.5)$ & $23(54.8)$ & $5(71.4)$ & .74 \\
\hline Crawford extent (I/II/III) & $19 / 40 / 56$ & $14 / 19 / 33$ & $5 / 19 / 18$ & $0 / 2 / 5$ & \\
\hline$\%$ Crawford extent II & 34.8 & 28.8 & 45.2 & 28.6 & .08 \\
\hline Marfan syndrome & $21(18.3)$ & $11(17.5)$ & $8(19.0)$ & $2(28.6)$ & .95 \\
\hline History of aortic surgery & $27(23.5)$ & $14(21.2)$ & $10(23.8)$ & $3(42.9)$ & .75 \\
\hline Emergency/urgent surgery & $15(13.0)$ & $11(16.7)$ & $4(9.5)$ & 0 & .45 \\
\hline Smoking history & $65(56.5)$ & $33(50.0)$ & $28(66.7)$ & $4(57.1)$ & .09 \\
\hline Hypertension & $93(80.9)$ & $51(77.3)$ & $35(83.3)$ & $6(85.7)$ & .60 \\
\hline Diabetes mellitus & $13(11.3)$ & $6(9.1)$ & $5(11.9)$ & $1(14.3)$ & .88 \\
\hline Hyperlipidemia & $26(22.6)$ & $12(18.2)$ & $11(26.2)$ & $2(28.6)$ & .32 \\
\hline Chronic renal failure $(\mathrm{sCr} \geq 2.0)$ & $12(10.4)$ & $7(10.6)$ & $4(9.5)$ & $1(14.3)$ & .88 \\
\hline Chronic obstructive pulmonary disease & $10(8.7)$ & $5(7.6)$ & $5(11.9)$ & 0 & \\
\hline Permanent hemodialysis & $6(5.2)$ & $3(4.5)$ & $3(7.1)$ & 0 & .89 \\
\hline CSF drainage & $95(82.6)$ & $52(78.8)$ & $36(85.7)$ & $7(100)$ & .52 \\
\hline Preoperative AKA detection & $85(73.9)$ & $44(66.7)$ & $34(81.0)$ & $7(100)$ & .16 \\
\hline \multicolumn{6}{|l|}{ Timing of surgery } \\
\hline $1999-2006$ & $53(46.1)$ & $50(75.8)$ & $3(7.1)$ & 0 & $<.01$ \\
\hline $2007-2012$ & $62(53.9)$ & $16(24.2)$ & 39 (92.9) & $7(100)$ & $<.01$ \\
\hline
\end{tabular}
as much as possible to reduce the area of the aneurysmal wall.

\section{Data Definitions}

We defined SCII as any new lower-extremity motor or sensory deficit, or as both in the absence of any documented intracerebral hemispheric events. Patients who were fully ambulatory before surgery had to be able to bear

TABLE 1. Preoperative profiles of patients

$A K A$, Adamkiewicz artery; $C S F$, cerebrospinal fluid; $s C r$, serum creatinine; $S D$, standard deviation. ${ }^{*} P$ value means graft interposition versus aortic patch anastomosis. 


\section{Graft interposition}

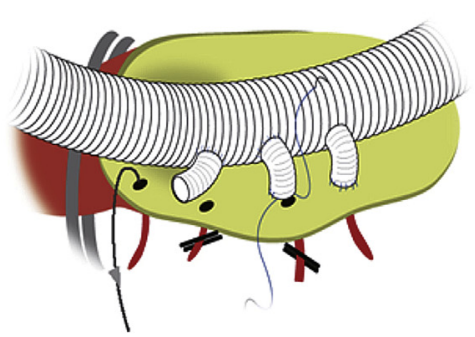

Aortic patch anastomosis

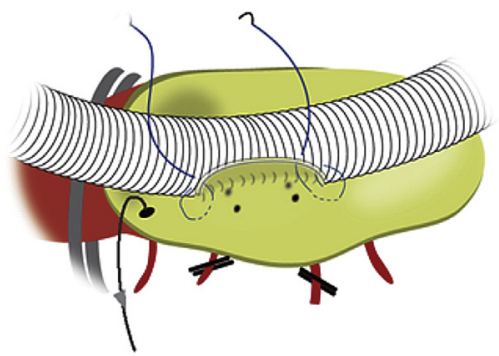

FIGURE 1. Techniques of graft interposition and aortic patch anastomosis. Graft interposition: Patent orifices of ICAs are reattached through a short graft ( 8 or $10 \mathrm{~mm}$ ) with an end-to-side anastomosis. Aortic patch anastomosis: Patent orifices of ICAs are anastomosed to a side hole in the graft as an aortic cuff using the inclusion technique.

their body weight without assistance to be neurologically intact. We considered the SCII to be transient when a clear documented deficit was fully resolved at the time of discharge. When symptoms had improved but functional status was not completely restored to preoperative levels, the complication was considered permanent. Early-onset SCII was defined as symptoms that became detectable when the patient awoke from anesthesia. Late-onset SCII was defined as spinal cord-related symptoms that appeared within 24 hours of completing the surgery.

\section{Postoperative Evaluation}

We routinely evaluated the postoperative patency of reconstructed ICAs using CT with contrast infusion $(n=104)$ or angiography $(n=11)$, starting from at least within 14 days after the surgery. The mean interval between surgery and postoperative evaluation was $8 \pm 3$ (range, 1-14) days. The patency of reconstructed ICAs was defined from CT findings by a radiologist at Kobe University with expertise in aortic imaging. Figure 2 shows representative patients in whom ICA patency was confirmed by $\mathrm{CT}$.

\section{Follow-up}

Follow-up data, including survival, general health, aortic reoperation or rupture, and cause of death, were obtained from our outpatient clinic, through written or telephone contact with patients or relatives, or from local cardiologists. The mean follow-up period was $49 \pm 38$ (range, 2-147) months, and follow-up information was obtained for $99.1 \%$ of the patients (1 patient was lost to follow-up). Patients were usually followed up by CT at our outpatient clinic or at one of several neighboring hospitals from 6 to 12 months after hospital discharge and annually thereafter. One or more postoperative CT assessments beyond 1 year after surgery proceeded in 76 patients. The mean period between initial surgery and the most recent CT assessment was $54 \pm 35$ (range, 12-144) months. Significant dilation at the site of reattached ICAs was defined by $5 \mathrm{~mm}$ or more dilation in the axial view of the most recent CT findings compared with initial postoperative CT.

\section{Statistical Analysis}

All data were statistically analyzed using SPSS 11.0 software (SPSS Inc, Chicago, Ill). Continuous data are expressed as means \pm standard deviation. Categoric variables were analyzed using the chi-square test, and risk factors for SCII were evaluated using stepwise logistic regression analysis. Clinically relevant variables with a $P$ value less than .2 in univariate analysis for SCII were incorporated into multivariate models to identify significant independent factors for SCII. Odds ratio estimates are reported with $95 \%$ confidence intervals. Model performance was evaluated using the $\mathrm{C}$-index (area under the receiver operating characteristic curve) as a measure of model discrimination and the Hosmer-Lemeshow test as a measure of "goodness-of-fit." Late survival and freedom from aortic surgery were estimated using the Kaplan-Meier method.

\section{RESULTS \\ Intraoperative Outcomes}

Table 2 shows the intraoperative outcomes. The average number of reconstructed ICAs in all patients was $3.0 \pm 1.4$ (range, 1-7). The ICAs were reconstructed using graft interposition $(n=66)$, island reconstruction $(n=42)$, and by both methods $(\mathrm{n}=7)$. We reconstructed a total of 345 ICAs, of which 225 and 120 were achieved using graft interposition and island reconstruction, respectively. Island reconstruction included 1, 2, 3, and 4 ICAs in 45, 22, 7, and 3 patients, respectively. The mean durations of cardiopulmonary bypass, ICA reconstruction, and visceral organ perfusion were $180 \pm 72$ (range, 43-351), $46 \pm 25$ (range, 12-120), and $74 \pm 41$ (range, 31-178) minutes, respectively. Graft replacement included an AKA in 65 patients.

\section{Postoperative Results}

Table 3 shows the postoperative results. The overall 30-day and in-hospital mortality rates were $0.9 \%(n=1)$ and $7.8 \%(\mathrm{n}=9)$, respectively. The causes of death were respiratory failure $(\mathrm{n}=3)$, sepsis $(\mathrm{n}=3)$, cerebral bleeding $(\mathrm{n}=2)$, and mesenteric ischemia $(\mathrm{n}=1)$. The overall incidence of SCII was $9.6 \%(\mathrm{n}=11$ patients: Crawford extent I $[\mathrm{n}=2]$, II [n=1], and III [n=8]). Permanent and transient SCII developed in 6 patients $(5.2 \%)$ and 5 patients $(4.3 \%)$, but late onset SCII did not occur. Seven patients were returned to the operating room because of postoperative bleeding. The rates of tracheostomy and temporary hemodialysis were $13.0 \% \quad(15 / 115)$ and $13.9 \% \quad(16 / 115)$, respectively.

\section{Patency of Intercostal Arteries and Risk Factors for Spinal Cord Ischemic Injury}

Overall patency was $74.2 \%$ (256/345) and was significantly lower after graft interposition than after aortic patch 


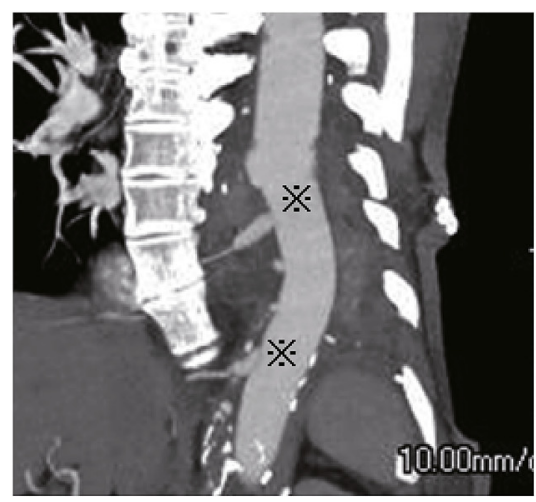

A

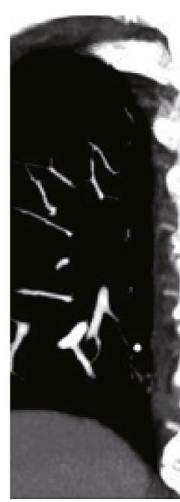

B
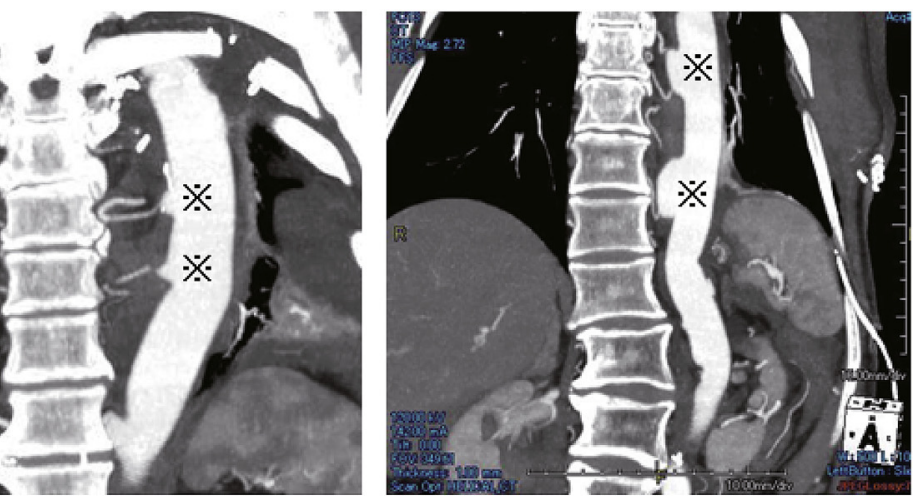

C

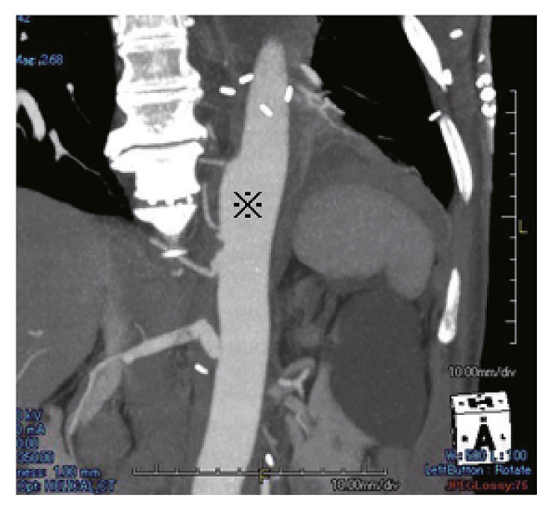

D

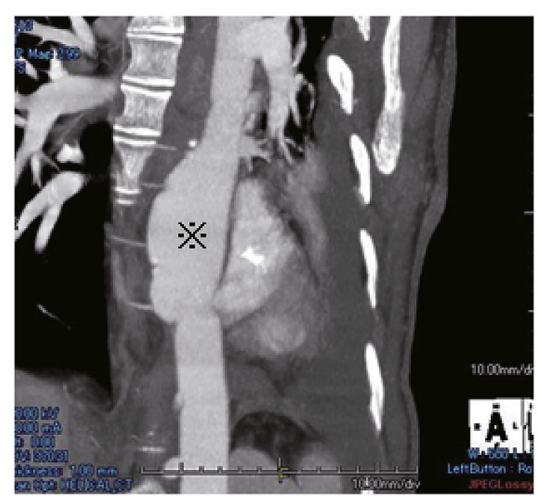

E

FIGURE 2. Postoperative findings of CT. A, Graft interposition. B-E, Aortic patch anastomosis, including 1 (B) $\times 2,2$ (C) $\times 2,3$ (D), and 4 (E) ICAs. *Sites of ICA reconstructions.

anastomosis $(65.3 \% \quad[147 / 225]$ vs $90.8 \% \quad$ [109/120], $P<.01)$. The patency of aortic patch anastomosis that included 1, 2, 3, and 4 ICAs was 86.7\% (39/45), 90.5\% (38/42), 100\% (21/21), and 91.7\% (11/12), respectively. Graft patency was significantly inferior in patients with than without SCII $(51.9 \%$ [14/27] vs $76.1 \%$ [242/318]). Table 4 shows the results of the univariate and multivariate analyses of SCII. Multivariate analysis significantly associated SCII with a patency rate of less than $50 \%$ (odds ratio, $10.0 ; P=.005)$. The $\mathrm{C}$-index was 0.96 for SCII in this univariate model (patency rate $<50 \%$ and SCII) and 0.75 in the multivariate model (including, age, nondissection, Crawford extent II and III, chronic obstructive pulmonary disease, and patency rate $<50 \%$ ). The Hosmer-Lemeshow test of the univariate and multivariate data showed $P=.91$ (patency rate $<50 \%$ and SCII) and .31 (including, age, nondissection, Crawford extent II and III, chronic obstructive pulmonary disease, and patency rate $<50 \%$ ), respectively.

To examine the association between the method of ICA reimplantation and SCII, we included the method of ICA reimplantation in the univariate model. This model comprised 108 patients (115-7 cases) because 7 patients underwent 2 procedures (graft interposition and aortic patch anastomosis). Univariate analysis showed that aortic patch anastomosis was not significantly associated with SCII (odds ratio, 0.35; 95\% confidence interval, 0.07-1.75; $P=.20)$.

\section{Late Results}

Among 115 patients, 106 were hospital survivors and 15 died during $49 \pm 38$ (range, 2-147) months of follow-up. Five- and 10 -year survivals were $85.7 \% \pm 3.9 \%$ and $63.3 \% \pm 10.7 \%$, respectively. No surgery was needed for aneurysm formation at the reconstructed ICAs. Nine additional aortic surgeries included the aortic root $(\mathrm{n}=2)$, ascending aorta $(\mathrm{n}=1)$, thoracic aorta $(\mathrm{n}=3)$, descending aorta $(\mathrm{n}=2)$, and abdominal aorta $(\mathrm{n}=1)$. Overall freedom from all aortic surgeries at 5 and 10 years was $87.8 \% \pm$ $4.6 \%$ and $82.1 \% \pm 5.8 \%$, respectively. Of the 106 hospital survivors, $76(71.7 \%)$ who were assessed by a minimum of 2 postoperative CT scans at least 1 year apart were amenable to growth rate analysis. We used CT to monitor $71.7 \%$ of patients $(43 / 60)$ with graft interposition and $71.7 \%$ of patients (33/46) with reconstructions using aortic patch anastomosis $(P=.99)$. The mean time from surgery to the most recent CT scans was $54 \pm 35$ (range, 12-144) months overall, $65 \pm 34$ (range, 12-125) months for graft interposition, and $39 \pm 30$ (range 12-144) months for aortic 
TABLE 2. Intraoperative outcomes

\begin{tabular}{|c|c|c|c|c|c|}
\hline Variables & Overall $(\%)$ & Graft interposition $(\%)$ & Aortic patch anastomosis (\%) & Both methods (\%) & $P$ value* \\
\hline Average reconstructed ICAs per case & $3.0 \pm 1.4(1-7)$ & $3.2 \pm 1.6(1-7)$ & $2.5 \pm 1.1(1-5)$ & $3.7 \pm 0.8(3-5)$ & $<.01$ \\
\hline No. of ICA reconstructions & 345 & 213 & 106 & 26 & \\
\hline No. of graft interpositions & 225 & 213 & 0 & 12 & \\
\hline \multicolumn{6}{|l|}{ Range of island reconstructions } \\
\hline $1 \mathrm{ICA}$ & 45 & N/A & 41 & 4 & \\
\hline 2 ICAs & 21 & N/A & 19 & 2 & \\
\hline 3 ICAs & 7 & N/A & 5 & 2 & \\
\hline 4 ICAs & 3 & N/A & 3 & & \\
\hline Duration of cardiopulmonary bypass & $180 \pm 72$ & $180 \pm 75$ & $176 \pm 65$ & $210 \pm 85$ & .81 \\
\hline Duration of ICAs reconstruction & $46 \pm 25$ & $60 \pm 40$ & $33 \pm 15$ & $71 \pm 51$ & $<.01$ \\
\hline Duration of visceral organ perfusion & $74 \pm 41$ & $77 \pm 45$ & $67 \pm 29$ & $116 \pm 45$ & .26 \\
\hline Deep hypothermia & $37(32.1)$ & $24(36.4)$ & $12(28.6)$ & $1(14.3)$ & .40 \\
\hline Range of graft replacements including AKA & $65(56.5)$ & $33(50.0)$ & $25(59.5)$ & $7(100)$ & .33 \\
\hline No. of transfused red cells (unit) & $25 \pm 19$ & $27 \pm 22$ & $21 \pm 10$ & $28 \pm 22$ & .14 \\
\hline
\end{tabular}

$A K A$, Adamkiewicz artery; $I C A$, intercostal artery; $N / A$, not available. $* P$ value means graft interposition versus aortic patch anastomosis.

patch anastomosis, respectively. The elapsed time from surgery to the most recent CT scan in graft interposition was significantly longer than in aortic patch anastomosis $(P<.01)$. Size expansion and false aneurysms were not found at sites of ICA reconstruction achieved by graft interposition or small areas of aortic patch anastomosis (including 1 or 2 ICAs). The ICA reconstruction site after aortic patch anastomosis became significantly dilated (from $35 \times 42$ to $38 \times 48 \mathrm{~mm}$ and from $44 \times 47$ to $49 \times 64 \mathrm{~mm}$ at 27 and 109 months postoperatively, respectively) in 2 patients whose aortic patch anastomosis included 4 ICAs. These patients remain under close biannual CT surveillance (Table 5).

\section{DISCUSSION}

Several technical improvements, such as distal aortic perfusion, CSF drainage, management using deep hypothermia, and intraoperative monitoring of the spinal cord, have been attempted to decrease SCII, which is one of the most serious complications of TAAA repair. ${ }^{3,7-9}$ Nevertheless, TAAA repair remains one of the most invasive types of aortic surgery. A recent report from Japan found a hospital mortality rate of $13.6 \%$ after TAAA repair. ${ }^{10}$ Although controversial, ICA reattachment has been considered one of the most effective strategies for preventing SCII, ${ }^{11-13}$ and several authors have reported that they reconstruct ICAs as much as possible. ${ }^{14,15}$ Although several surgical techniques have been described, the simplest method of reattaching ICAs is aortic patch anastomosis to a prosthetic graft. We have reconstructed the ICA using graft interposition, end-to-side anastomosis between a short graft ( 8 or $10 \mathrm{~mm}$ ), and by aortic patch anastomosis with the inclusion technique, creating a side-to-side anastomosis between the prosthetic graft and the aorta surrounding the intercostal vessels. Each of these methods has advantages and disadvantages.

Aortic patch anastomosis is relatively simple and can decrease the anastomosis site. However, the suture line can sometimes be too long and sutures can sometimes become loose. A fragile aortic wall can cause difficulties with bleeding control around an anastomosis site. Furthermore, late complications of aortic patch anastomosis reportedly include ICA patch aneurysms in which the retained aortic cuff becomes aneurysmal.

TABLE 3. Postoperative outcomes

\begin{tabular}{|c|c|c|c|c|c|}
\hline Variables & $\begin{array}{c}\text { Overall } \\
\mathbf{N}=115(\%)\end{array}$ & $\begin{array}{c}\text { Graft interposition } \\
n=66(\%)\end{array}$ & $\begin{array}{l}\text { Aortic patch anastomosis } \\
\mathrm{n}=\mathbf{4 2}(\%)\end{array}$ & $\begin{array}{c}\text { Both methods } \\
n=7(\%)\end{array}$ & $P$ value* \\
\hline 30-d mortality & $1(0.9)$ & $1(1.5)$ & 0 & 0 & .82 \\
\hline In-hospital mortality & $9(7.8)$ & $6(9.1)$ & $3(7.1)$ & 0 & 1.0 \\
\hline SCII & $11(9.6)$ & $8(12.1)$ & $2(4.8)$ & $1(14.3)$ & .34 \\
\hline Permanent & $6(5.2)$ & $4(6.1)$ & $2(4.8)$ & 0 & .89 \\
\hline Transient & $5(4.3)$ & $4(6.1)$ & 0 & $1(14.3)$ & .27 \\
\hline Early onset & $11(9.6)$ & $8(12.1)$ & $2(4.8)$ & $1(14.3)$ & .34 \\
\hline Late onset & 0 & 0 & 0 & 0 & \\
\hline Reexploration for bleeding & $7(6.1)$ & $4(6.1)$ & $2(4.8)$ & $1(14.3)$ & .89 \\
\hline Tracheostomy & $15(13.0)$ & $8(12.1)$ & $6(14.3)$ & $1(14.3)$ & .97 \\
\hline Temporary hemodialysis & $16(13.9)$ & $10(15.2)$ & $5(11.9)$ & $1(14.3)$ & .85 \\
\hline
\end{tabular}

$S C I I$, Spinal cord ischemic injury. $* P$ value means graft interposition versus aortic patch anastomosis. 
TABLE 4. Risk factors for spinal cord ischemic injury identified by univariate and multivariate analyses (revised version)

\begin{tabular}{|c|c|c|c|c|}
\hline \multirow[b]{2}{*}{ Variable } & \multicolumn{2}{|c|}{ Univariate } & \multicolumn{2}{|c|}{ Multivariate } \\
\hline & OR $(95 \%$ CI $)$ & $\boldsymbol{P}$ & OR $(95 \%$ CI $)$ & $P$ \\
\hline Age & $1.05(0.99-1.12)$ & .12 & $0.99(0.92-1.06)$ & .77 \\
\hline Aortic cause (nondissection) & $3.50(0.88-13.9)$ & .08 & $1.73(0.30-9.90)$ & .54 \\
\hline Crawford extent II & $0.17(0.02-1.35)$ & .09 & $0.63(0.03-12.8)$ & .76 \\
\hline Crawford extent III & $3.94(0.99-15.7)$ & .05 & $2.57(0.40-16.4)$ & .32 \\
\hline Emergency/urgent & $1.56(0.30-8.01)$ & .60 & & \\
\hline History of aortic surgery & $1.25(0.31-5.08)$ & .76 & & \\
\hline Chronic renal insufficiency $(\mathrm{sCr} \geq 2.0$ ) & $0.85(0.10-7.25)$ & .88 & & \\
\hline COPD & $5.20(1.12-24.1)$ & .04 & $3.05(0.46-20.0)$ & .25 \\
\hline CSF drainage & $2.24(0.27-18.5)$ & .46 & & \\
\hline Preoperative AKA detection & $1.66(0.34-8.15)$ & .53 & & \\
\hline Duration of cardiopulmonary bypass & $1.00(0.99-1.01)$ & .69 & & \\
\hline Duration of ICA reconstructions & $1.01(0.99-1.03)$ & .51 & & \\
\hline Deep hypothermia & $0.44(0.09-2.14)$ & .31 & & \\
\hline AKA reconstruction & $1.03(0.30-3.58)$ & .96 & & \\
\hline Patency rate $<50 \%$ & $10.0(2.49-40.1)$ & .001 & $10.0(2.02-49.7)$ & .005 \\
\hline
\end{tabular}

$\overline{A K A}$, Adamkiewicz artery; $C I$, confidence interval; $C O P D$, chronic obstructive pulmonary disease; $C S F$, cerebrospinal fluid; $I C A$, intercostal artery; $O R$, odds ratio; $s C r$, serum creatinine.

On the other hand, the diseased aortic cuff is smaller in graft interposition and is considered less risky than ICA patch aneurysms. Suture bleeding is easier to control, and spinal cord ischemic duration is shorter with concomitant sequential clamping. The major disadvantage is that this procedure requires more anastomosis and graft arrangement and is thus time-consuming and occasionally challenging.

Patency rates and relationships between patency and SCII, as well as ICA patch aneurysms, have not been reported in detail. The present study found that patency was worse for graft interposition than for aortic patch anastomosis. We previously reattached ICAs mainly by graft interposition because of the supposed advantages described, but ICA patency was significantly lower than that associated with aortic patch anastomosis. The reasons for the lower patency after graft interposition might be graft twisting, turbulence around the anastomosis site, the purse-string effect, or a mismatch between the anastomosis and the ICA orifice.

The patency rate of reattached ICAs in the present study was lower in patients with than without SCII. Univariate and multivariate analyses showed a significant relationship between patency rates less than $50 \%$ and SCII. The patency of reconstructed ICAs might be influenced by postoperative factors, such as the hemodynamic stability of the patient or the timing of imaging assessment. However, none of our patients developed late-onset SCII, and all SCIIs were detected when patients awoke from anesthesia. Thus, the number of possible postoperative factors was considered to be limited.

Univariate analysis did not uncover a significant correlation between AKA and SCII, perhaps because the spinal cord is perfused not only by the AKA but also by numerous collateral networks. ${ }^{16}$ However, the AKA is one of the main suppliers of blood to the spinal cord. The preoperative detection of an AKA could help surgeons to design surgical strategies regarding aortic clamp positioning or to determine which ICAs will require reconstruction during TAAA repair. ${ }^{17}$ The spinal cord might be protected more effectively if critical ICAs are reconstructed by methods that are likely to confer better patency.

In addition, univariate analysis showed that aortic patch anastomosis was not significantly associated with SCII. This finding suggested that the mode of ICA reconstruction alone cannot prevent SCII and supported the multidisciplinary approach that many authors advocate as being important to prevent SCII. No single method can prevent SCII with $100 \%$ certainty. We also believe that a multidisciplinary approach is essential to prevent SCII. That the mode of ICA reconstruction was not significant is a limitation of our study. Nonetheless, we found that the patency of

TABLE 5. The dilation of aortic patch anastomosis

\begin{tabular}{|c|c|c|c|c|c|c|c|}
\hline \multirow[b]{2}{*}{ Age } & \multirow[b]{2}{*}{ Sex } & \multirow{2}{*}{$\begin{array}{c}\text { Crawford } \\
\text { classification }\end{array}$} & \multirow[b]{2}{*}{ Marfan } & \multirow{2}{*}{$\begin{array}{c}\text { Aortic patch } \\
\text { anastomosis including }\end{array}$} & \multirow{2}{*}{$\begin{array}{c}\text { Time from initial } \\
\text { to latest } \mathbf{C T}\end{array}$} & \multicolumn{2}{|c|}{ Size at } \\
\hline & & & & & & Initial CT & Latest CT \\
\hline 42 & Male & II & Yes & 4 ICAs & 27 & $35 \times 42$ & $38 \times 48$ \\
\hline 59 & Female & I & No & 4 ICAs & 109 & $44 \times 47$ & $49 \times 64$ \\
\hline
\end{tabular}

CT, Computed tomography; ICA, intercostal artery. 
reattached ICAs was significantly better after aortic patch anastomosis than graft interposition, and that the less than $50 \%$ patency rate of reattached ICAs was significantly associated with SCII. We believe that ICAs should be reconstructed by a method that can deliver better patency, and that this benefits spinal cord circulation.

Zoli and colleagues ${ }^{18}$ reported that the number of sacrificed ICAs was the most powerful predictor of SCII. When 13 or more segmental arteries were sacrificed, the risk of SCII increased from $1.2 \%(<8$ ICAs being sacrificed) to $12.5 \%$. This finding suggested that spinal cord perfusion that is mainly dependent on collateral pathways is dangerous during extensive TAAA graft replacement without ICA reconstruction. We believe that ICA reattachment is essential to prevent SCII in patients with extensive TAAA, such as Crawford extent II.

Aneurysms of ICA patches must be closely monitored as late complications of TAAA repair. Kulik and coworkers ${ }^{5}$ described 11 ICA patch aneurysms that developed after TAAA repair. Half of their patients had Marfan syndrome. The incidence rate of ICA patch aneurysm was $7.1 \%$ during 22 years of follow-up, and reintervention for ICA patch aneurysms arising from initial operation was 5.3 years in their study. Dardik and associates ${ }^{19}$ described 8 patients with visceral patch aneurysms, 3 of whom had connective tissue disorders. The incidence rate of visceral patch aneurysms was $7.5 \%$ over 8 years, and the mean time to detection was 6.5 years after the original operation. All ICA and visceral branches in the reports described were reconstructed using aortic patch anastomosis. A connective tissue disorder seemed to be a risk factor for late aneurysmal changes at sites of ICA reconstructions. The ICA patch became dilated at the site of aortic patch anastomosis after 27 and 109 months in 2 of our patients from $35 \times 42$ to $38 \times 48 \mathrm{~mm}$ and from $44 \times 47$ to $49 \times 64 \mathrm{~mm}$, respectively. These patients underwent 4 consecutive ICA reconstructions from Th9-11. On the other hand, false or patch aneurysms after graft interposition and small areas of aortic patch anastomosis (including 1 or 2 ICAs) were undetectable in our patients. These results suggested that the risk of aneurysm formation is greater when large blocks of aortic wall are reconstructed. To prevent late dilation, the patch area of ICA anastomoses should be minimized.

\section{Study Limitations}

The study design was a single-institution retrospective study with a small number of patients. A randomized comparison of early patency between 2 methods was difficult to conduct. Preoperative patient characteristics and technical learning curves can influence early patency. Not all patients were assessed by $\mathrm{CT}$ after the initial operation and during follow-up. The observational duration was relatively short and differed between patients treated with graft interposition and aortic patch anastomosis.

\section{CONCLUSIONS}

Aortic patch anastomosis may be more appropriate than graft interposition because the patency rate is better and spinal cord ischemia might be prevented. Although aneurysmal changes at ICA reconstruction sites were rare, patients who underwent large blocks of aortic wall reconstruction require close follow-up.

\section{References}

1. Wong DR, Parenti JL, Green SY, Chowdhary V, Liao JM, Zarda S, et al. Open repair of thoracoabdominal aortic aneurysm in the modern surgical era; contemporary outcomes in 509 patients. J Am Coll Surg. 2011;212:569-79.

2. Schepens MA, Heijmen RH, Ranshaert W, Sonker U, Morshuis WJ. Thoracoabdominal aortic aneurysm repair; results of conventional open surgery. Eur J Vasc Endovasc Surg. 2009;37:640-5.

3. Kulik A, Castner CF, Kouchoukos NT. Outcomes after thoracoabdominal aortic aneurysm repair with hypothermic circulatory arrest. J Thorac Cardiovasc Surg. 2011;141:953-60.

4. Etz CD, Halstead JC, Spielvogel D, Shahani R, Lazala R, Homann TM, et al. Thoracic and thoracoabdominal aneurysm repair: is reimplantation of spinal cord arteries a waste of time? Ann Thorac Surg. 2006;82:1670-7.

5. Kulik A, Allen BT, Kouchoukos NT. Incidence and management of intercostal patch aneurysms after repair of thoracoabdominal aortic aneurysms. $J$ Thorac Cardiovasc Surg. 2009;138:352-8.

6. Okita Y, Omura A, Yamanaka K, Inoue T, Kano H, Tanioka R, et al. Open reconstruction of thoracoabdominal aortic aneurysms. Ann Cardiothorac Surg. 2012; 1:373-80.

7. Safi HJ, Miller CC, Huynn TT, Estrera AL, Porat EE, Winnerkvist AN, et al Distal aortic perfusion and cerebrospinal fluid drainage for thoracoabdominal and descending thoracic aortic repair: ten years of organ protection. Ann Surg. 2003;238:372-80.

8. Coselli JS, Lemaire SA, Koksoy C, Schmitting ZC, Curling PE. Cerebrospinal fluid drainage reduces paraplegia after thoracoabdominal aortic aneurysm repair: results of a randomized clinical trial. J Vasc Surg. 2002;35:631-9.

9. Jacobs MJ, Mess W, Mochtar B, Nijenhuis RJ, Statius van Eps RG, Schurink GW. The value of motor evoked potentials in reducing paraplegia during thoracoabdominal aneurysm repair. J Vasc Surg. 2006;43:239-46.

10. Kuwano H, Amano J, Yokomise H. Thoracic and cardiovascular surgery in Japan during 2010; Annual report by the Japanese Association for Thoracic Surgery. Gen Thorac Cardiovasc Surg. 2012;10:680-708.

11. Safi HJ, Miller CC, Carr C, Iliopoulos DC, Dorsay DA, Baldwin JC. Importance of intercostal artery reattachment during thoracoabdominal aortic aneurysm repair. J Vasc Surg. 1998;27:58-66.

12. Kuniyoshi Y, Koja K, Miyage K, Shimoji M, Uezu T, Arakaki K, et al. Prevention of postoperative paraplegia during thoracoabdominal aortic surgery. Ann Thorac Surg. 2003;76:1477-84.

13. Acher CW, Wynn MM, Mell MW, Tefera G, Hoch JR, et al. A quantitative assessment of the impact of intercostal artery reimplantation on paralysis risk in thoracoabdominal aortic aneurysm repair. Ann Surg. 2008;248:529-40.

14. Woo EY, McGarvey M, Jackson BM, Bavaria JE, Faiman RM, Pochettino A. Spinal cord ischemia may be reduced via a novel technique of intercostal artery revascularization during open thoracoabdominal aneurysm repair. $J$ Vasc Surg. 2007;46:421-6.

15. Mutsuga M, Narita Y, Araki Y, Maekawa A, Oshima H, Usui A. Spinal cord protection during a thoracoabdominal aortic repair for a chronic type B aortic dissection using the aortic tailoring strategy. Interact Cardiovasc Thorac Surg. 2010;11:15-9.

16. Griepp RB, Griepp EB. Spinal cord perfusion and protection during descending thoracic and thoracoabdominal aortic surgery; the collateral network concept. Ann Thorac Surg. 2007;83:S865-9.

17. Ogino H, Sasaki H, Minatoya K, Matsuda H, Yamada N, Kiamura S. Combined use of Adamkiewicz artery demonstration and motor-evoked potentials in descending and thoracoabdominal repair. Ann Thorac Surg. 2006;82:592-6.

18. Zoli S, Roder F, Etz CD, Brenner RM, Bodian CA, Lin HM. Predicting the risk of paraplegia after thoracic and thoracoabdominal aneurysm repair. Ann Thorac Surg. 2010;90:1237-44.

19. Dardik A, Perler BA, Roseborough GS, Williams GM. Aneurysmal expansion of the visceral patch after thoracoabdominal aortic replacement: an argument for limiting patch size? J Vasc Surg. 2001;34:405-9. 